\title{
Platinum single atoms supported on nanoarray-structured nitrogen-doped graphite foil with enhanced catalytic performance for hydrogen evolution reaction
}

Jingsong Xu, ${ }^{a_{\star}}$ Rui Li, ${ }^{b_{\sharp}}$ Rongguang Zeng, ${ }^{b}$ Xiayan Yan, ${ }^{b}$ Qingkai Zhao, ${ }^{b}$ Jingwen

Ba, ${ }^{b}$ Wenhua Luo, ${ }^{b *}$ and Daqiao Meng ${ }^{a *}$

a Science and Technology on Surface Physics and Chemistry Laboratory, Jiangyou,

Sichuan 621908, China.

b Institute of Materials, China Academy of Engineering Physics, Jiangyou, Sichuan 621907, China.

Supporting Figures

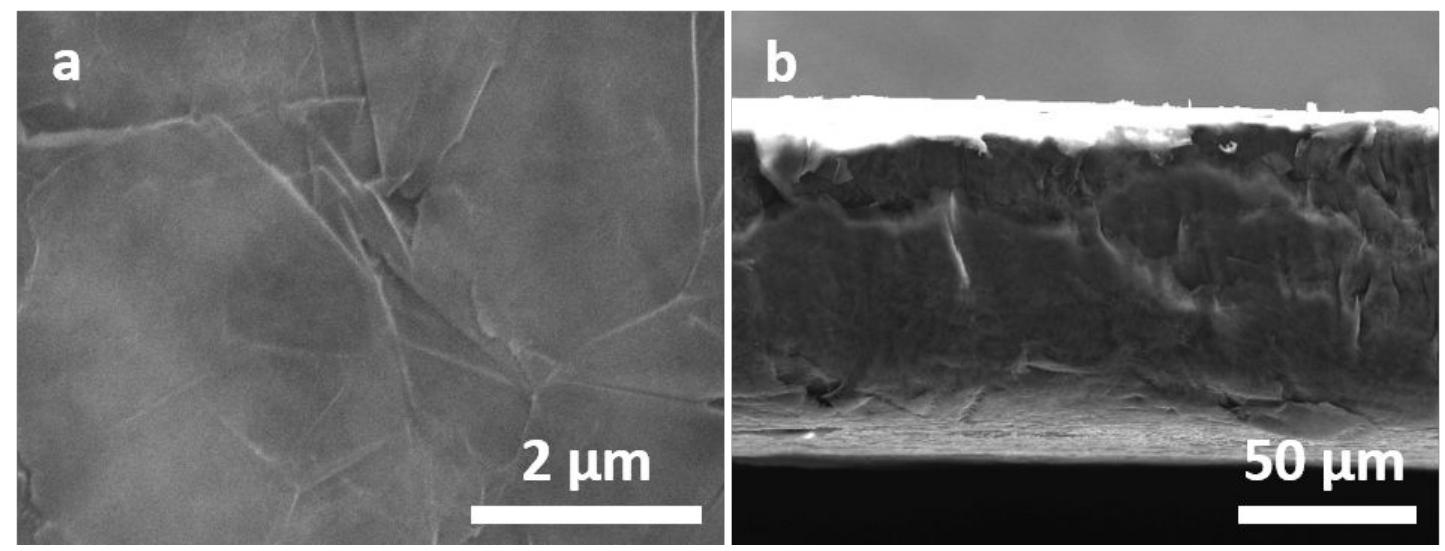

Figure S1. (a) Top-view and (b) cross-sectional SEM images of GF. 

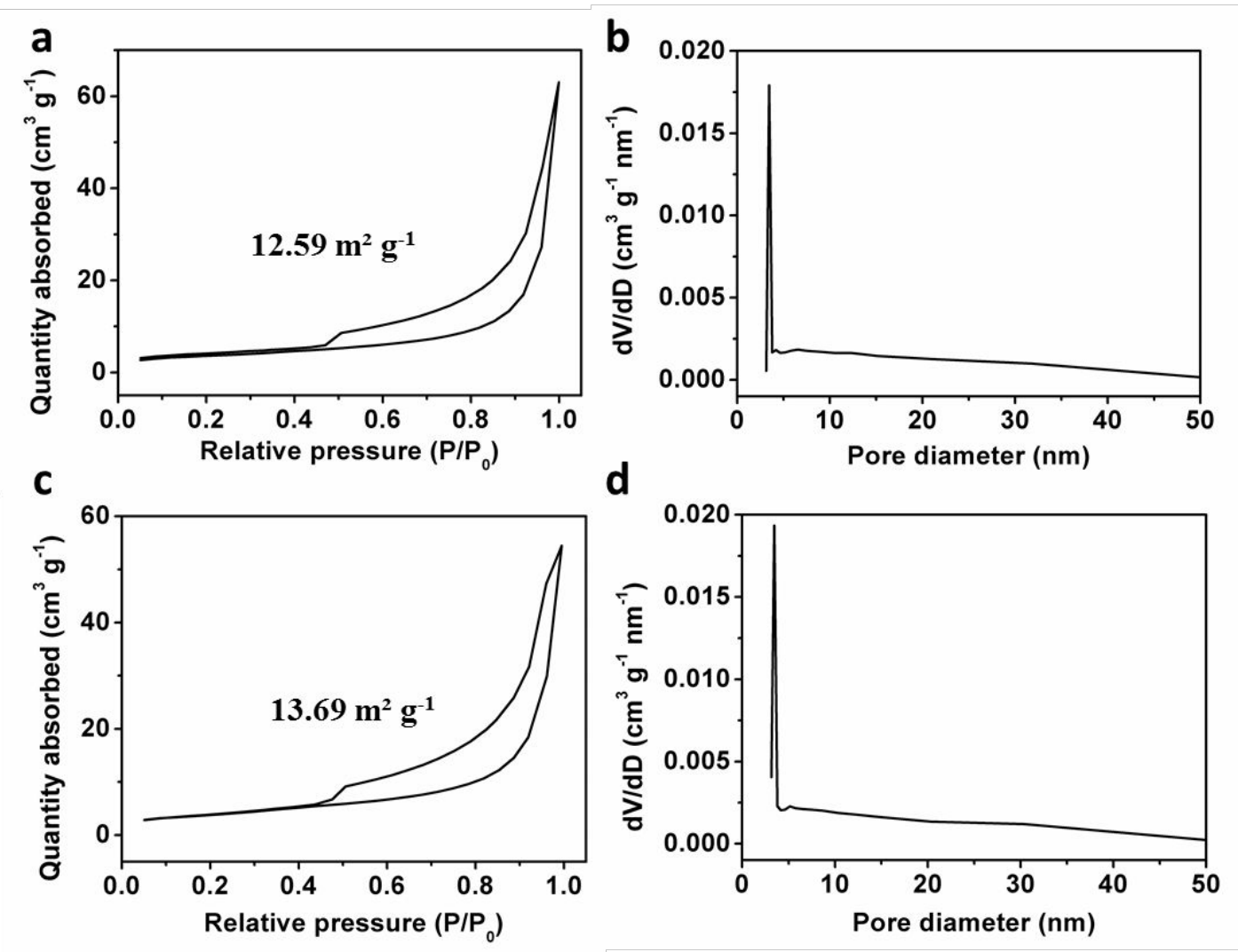

Figure S2. (a) The nitrogen adsorption-desorption isotherm curve and (b) the pore size distribution of GF; (c) the nitrogen adsorption-desorption isotherm curve and (d) the pore size distribution of NNGF.
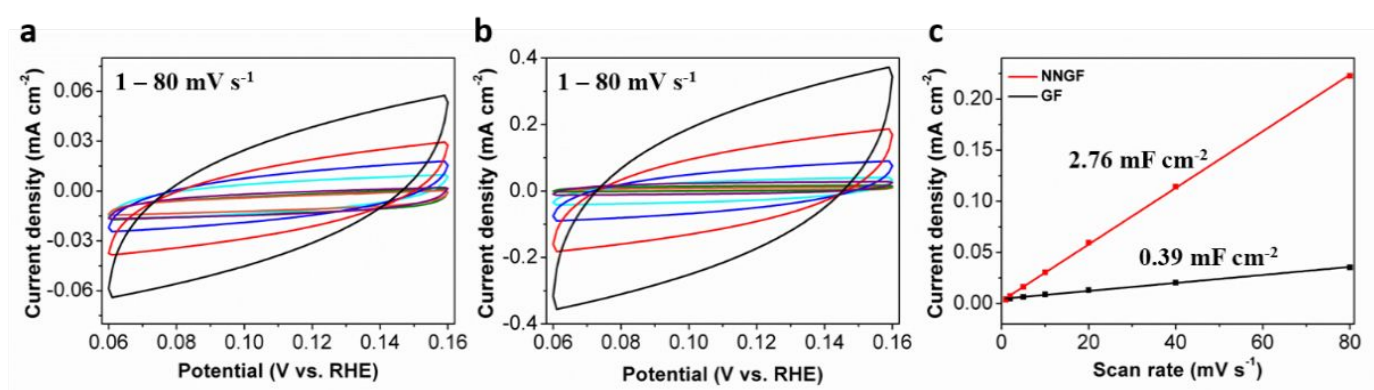

Figure S3. CVs of (a) GF and (b) NNGF electrodes at different scan rates of 1-80 $\mathrm{mV} \mathrm{s}^{-1}$ in the potential range of $0.06-0.16 \mathrm{~V}$ vs RHE (d) Capacitive current at $0.11 \mathrm{~V}$ vs RHE as a function of scan rates for GF and NNGF electrodes. 


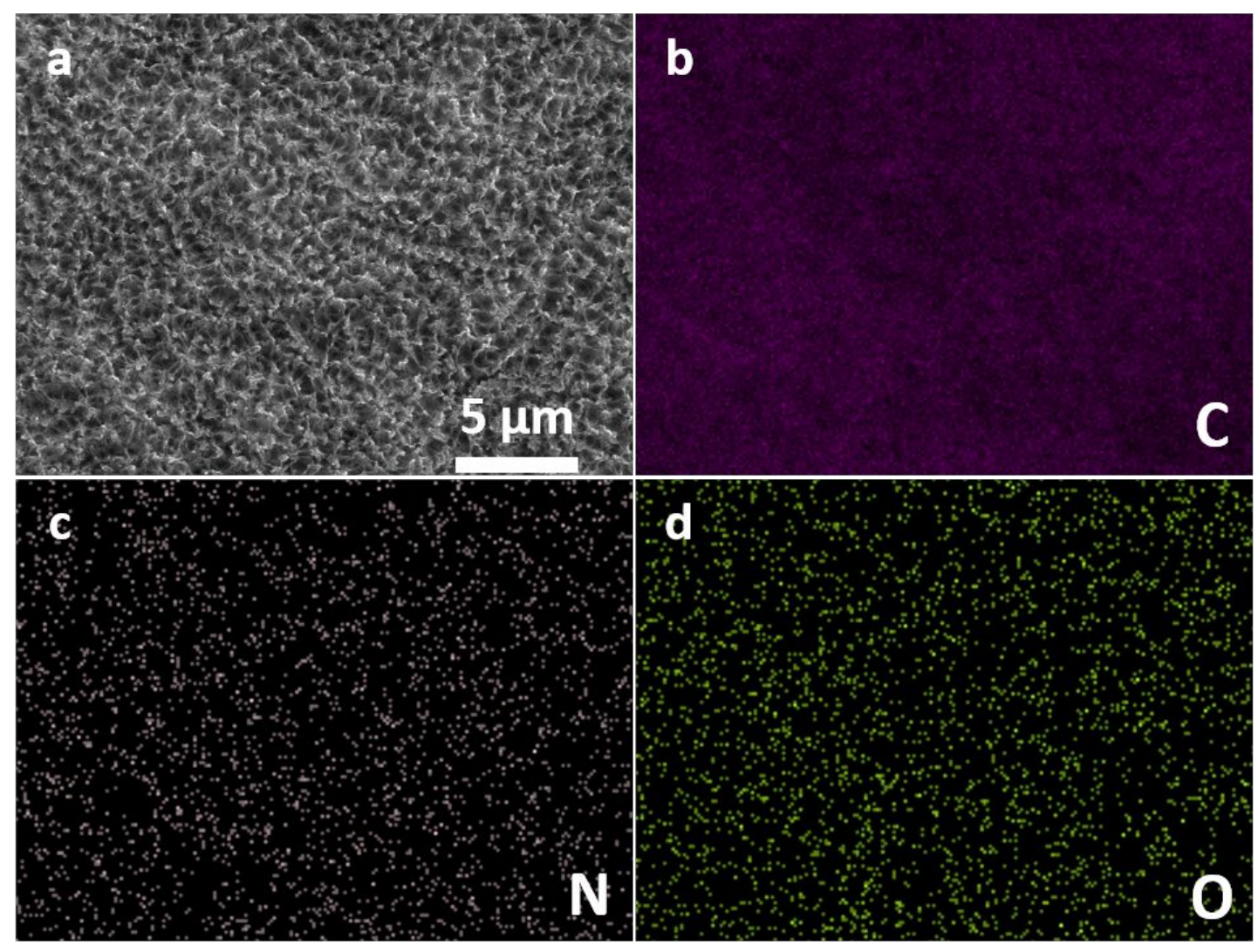

Figure S4. (a) SEM image and (b-d) its corresponding EDS elemental mappings of NNGF. 


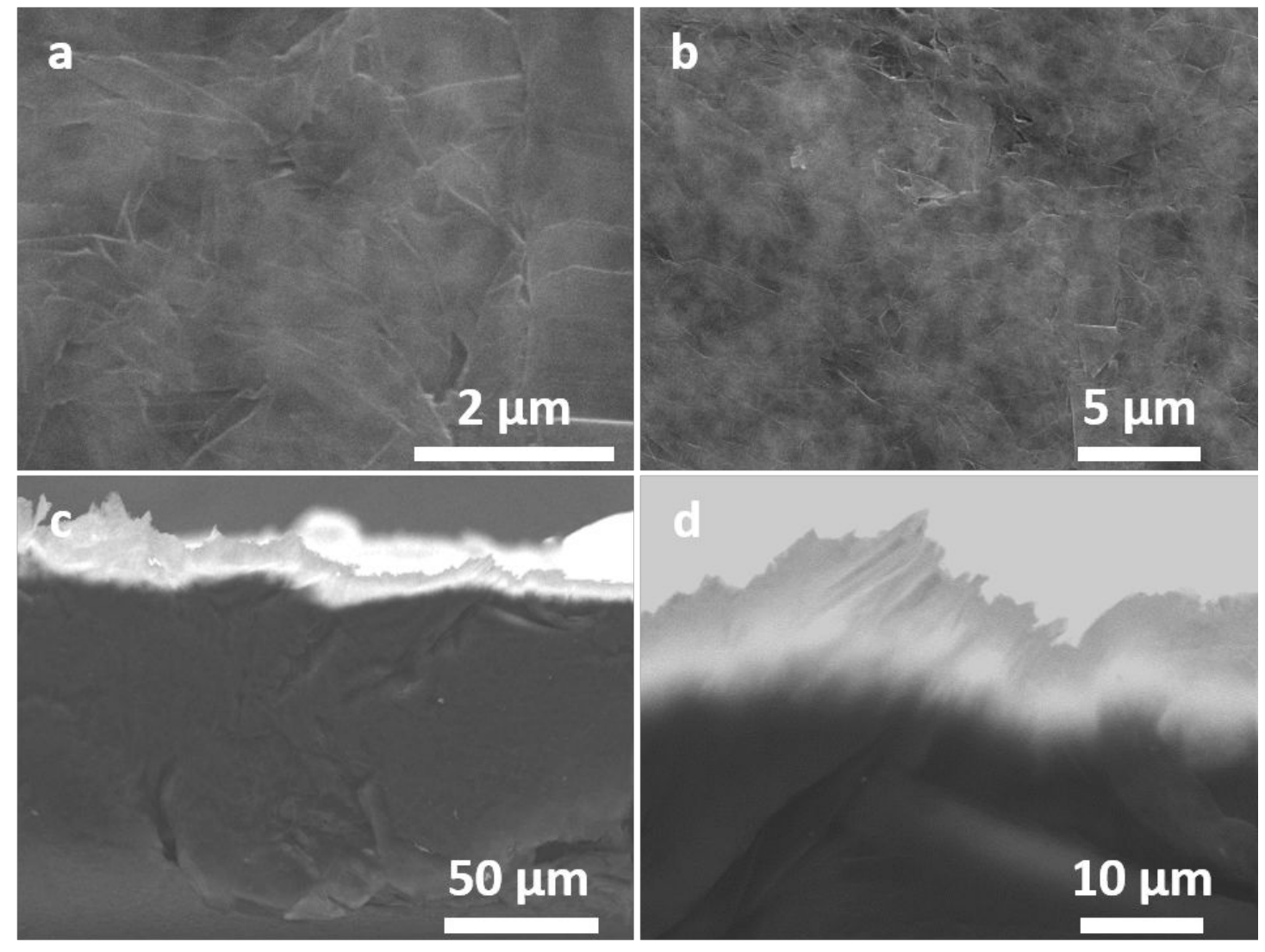

Figure S5. (a, b) SEM images of the bulk layer of NNGF; and (c, d) cross-sectional SEM images of NNGF.

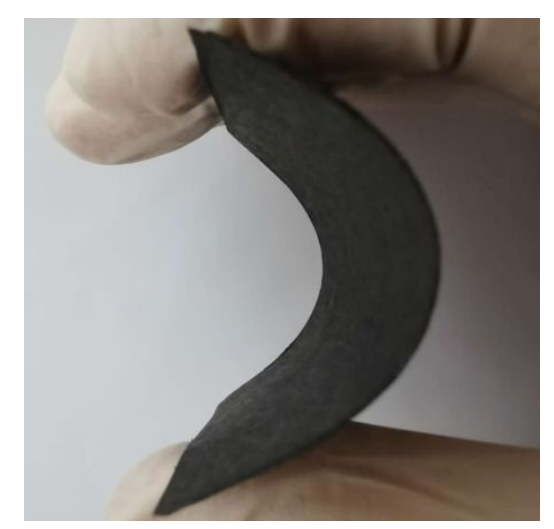

Figure S6. Digital pictures of flexible NNGF. 


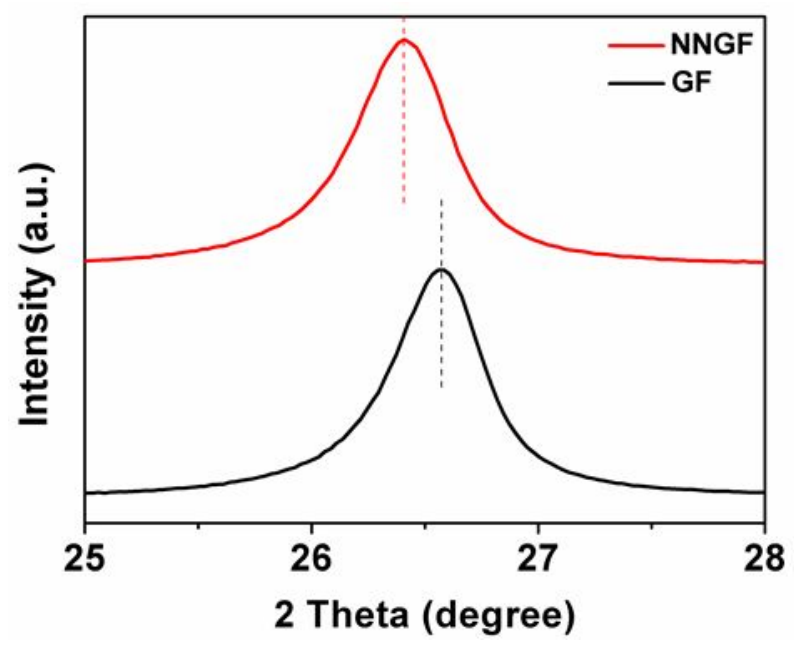

Figure S7. Enlarged XRD patterns of NNGF and GF.

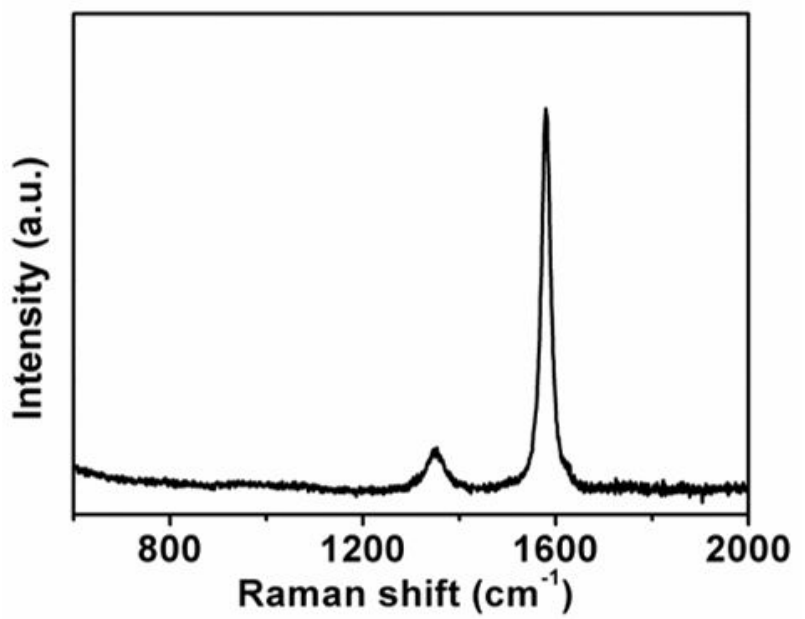

Figure S8. Raman spectrum of the bulk layer of NNGF. 

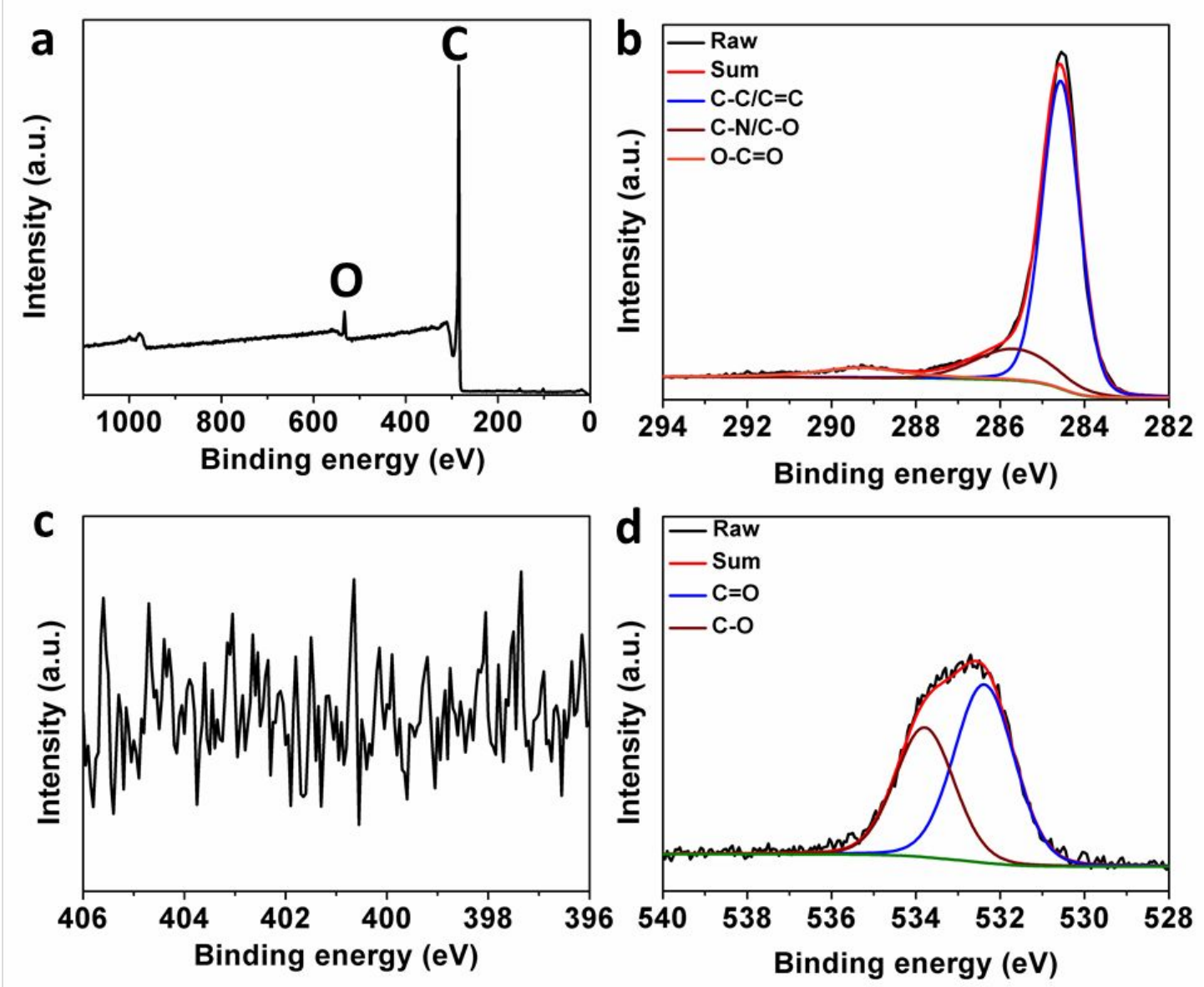

Figure S9. (a) XPS survey spectrum and high resolution (b) C 1s, (c) N 1s and (d) O 1s spectrum of GF.
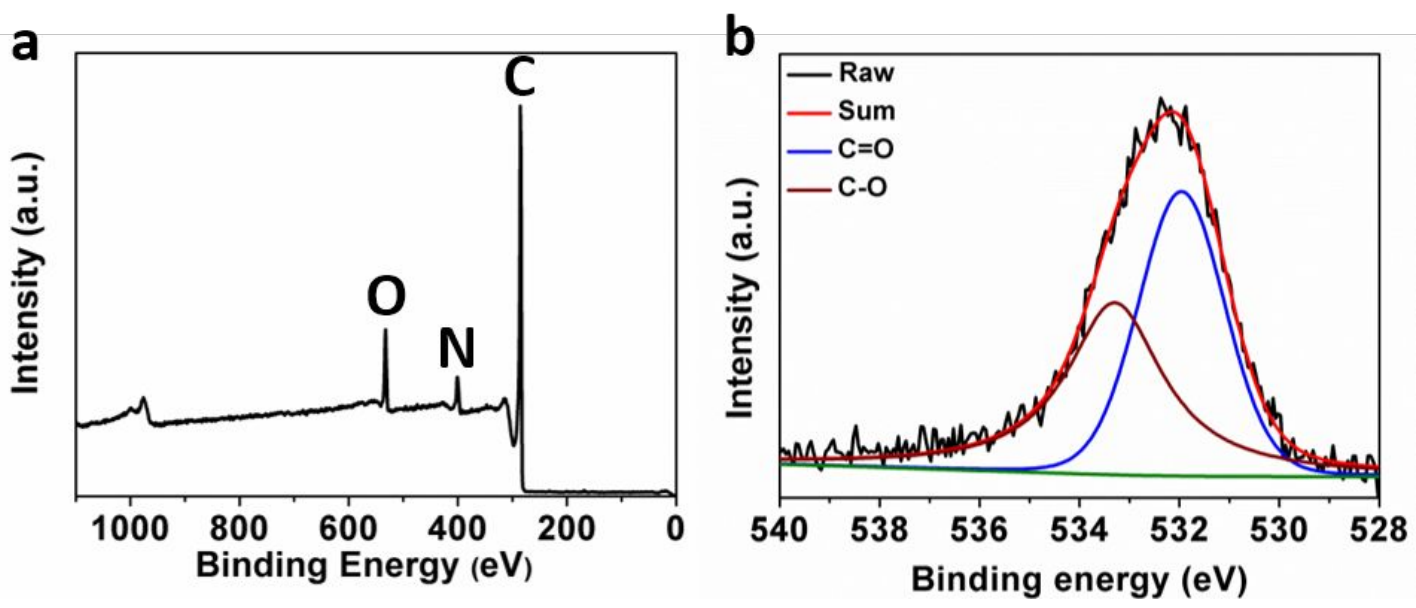

Figure S10. (a) XPS survey spectrum and (b) high resolution O 1s spectrum of NNGF. 


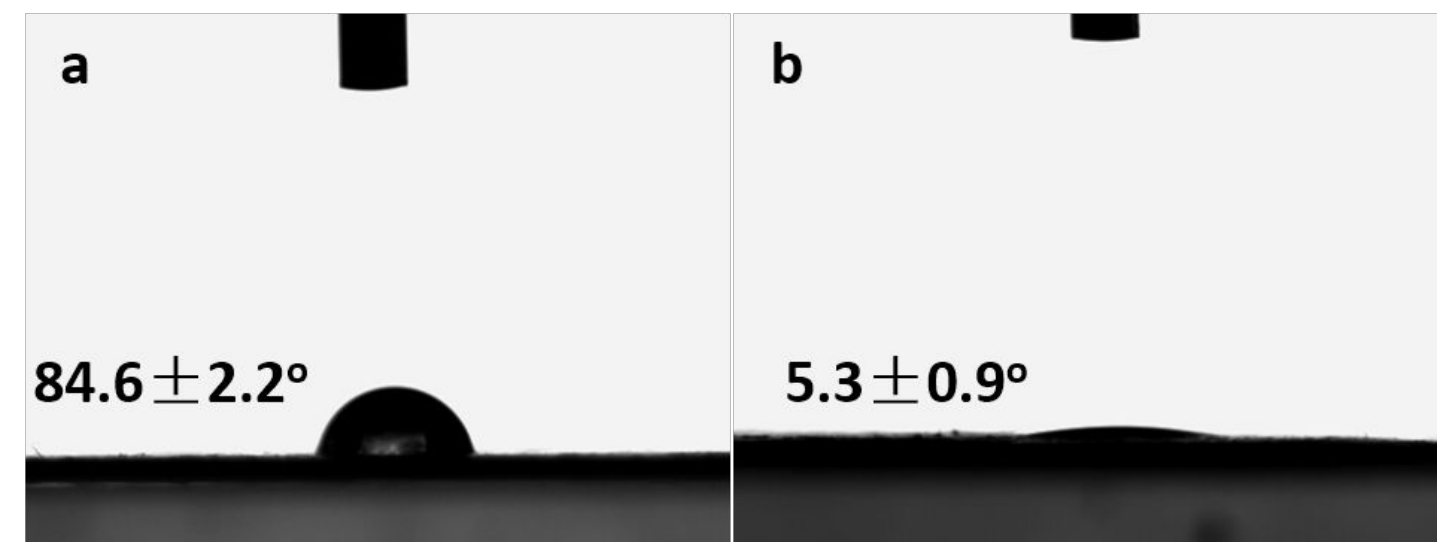

Figure S11. Water contact angle measurements for (a) GF and (b) NNGF.
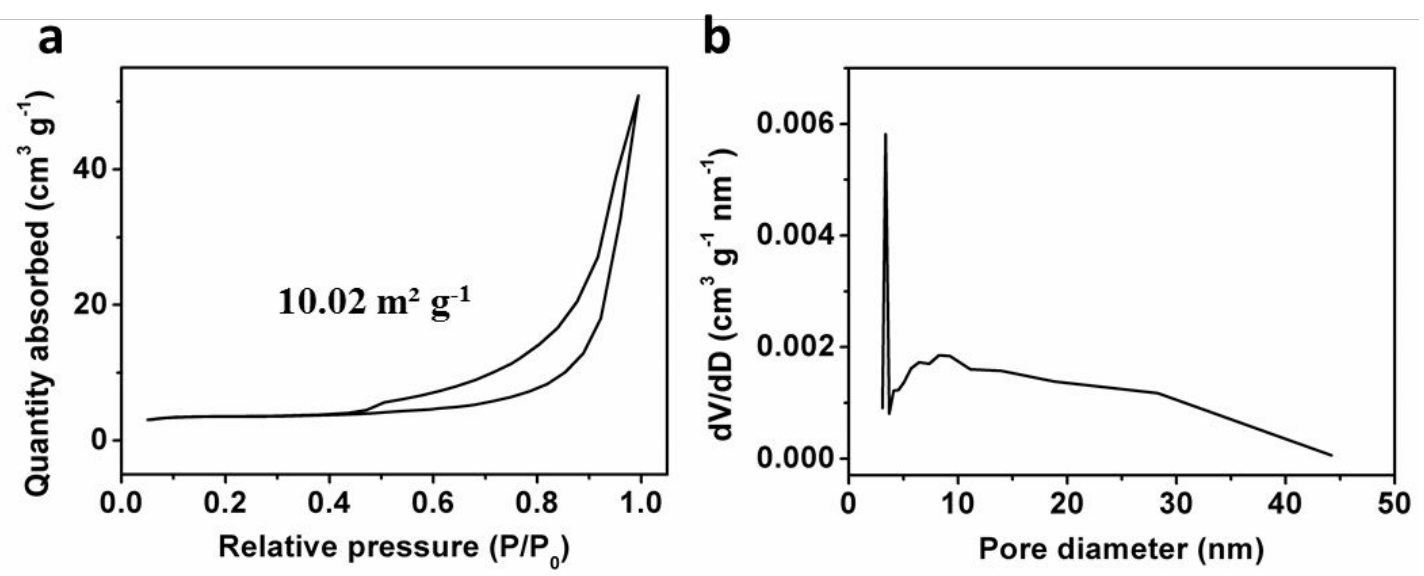

Figure S12. (a) The nitrogen adsorption-desorption isotherm curve and (b) the pore size distribution of $\mathrm{Pt}_{1} / \mathrm{NNGF}$.

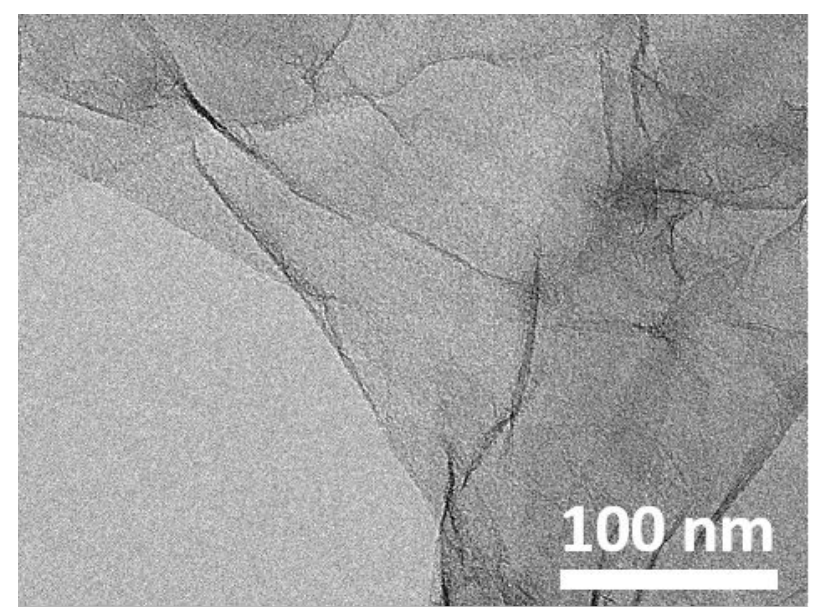

Figure S13. TEM images of $\mathrm{Pt}_{1} / \mathrm{NNGF}$. 


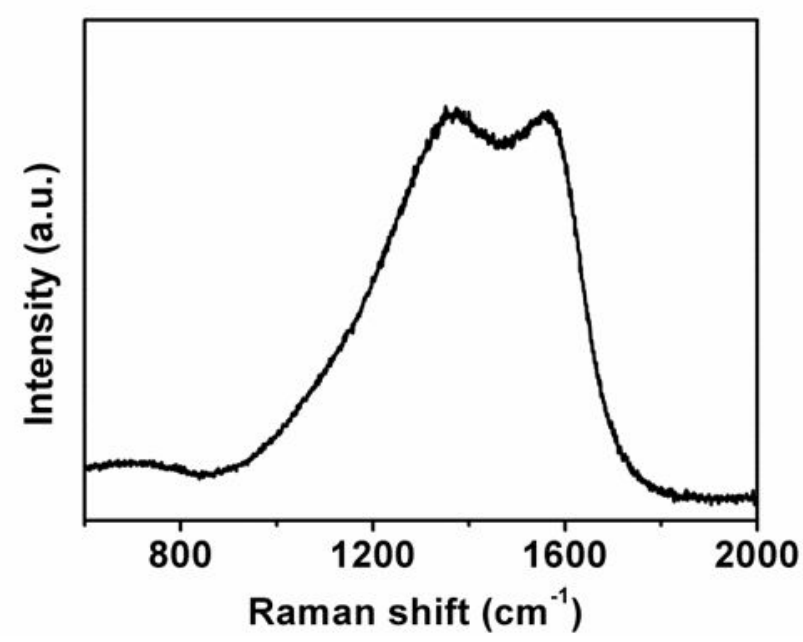

Figure S14. Raman spectrum of $\mathrm{Pt}_{1} / \mathrm{NNGF}$.
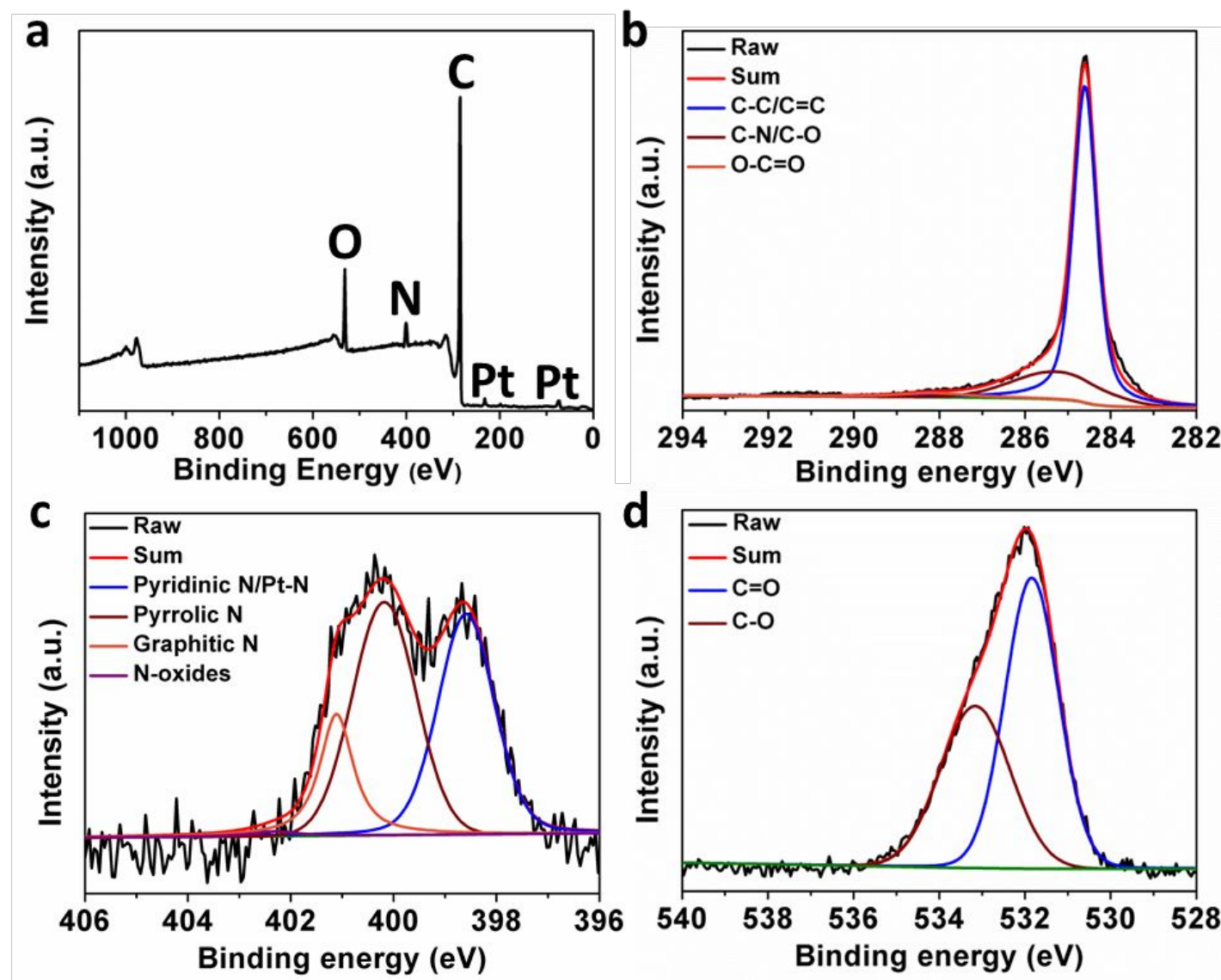

Figure S15. (a) XPS survey spectrum and high resolution (b) C 1s, (c) N 1s, and (d) O 1s spectra of $\mathrm{Pt}_{1} / \mathrm{NNGF}$. 

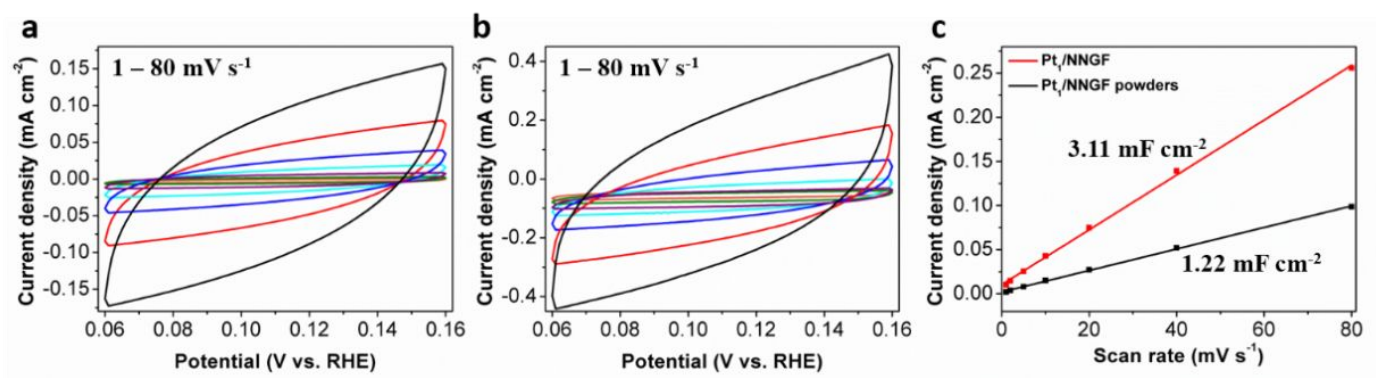

Figure S16. CVs of (a) $\mathrm{Pt}_{1} / \mathrm{NNGF}$ powder and (b) $\mathrm{Pt}_{1} / \mathrm{NNGF}$ electrodes at different scan rates of $1-80 \mathrm{mV} \mathrm{s}^{-1}$ in the potential range of $0.06-0.16 \mathrm{~V}$ vs RHE (d) Capacitive current at $0.11 \mathrm{~V}$ vs $\mathrm{RHE}$ as a function of scan rates for $\mathrm{Pt}_{1} / \mathrm{NNGF}$ powder and $\mathrm{Pt}_{1} / \mathrm{NNGF}$ electrodes.
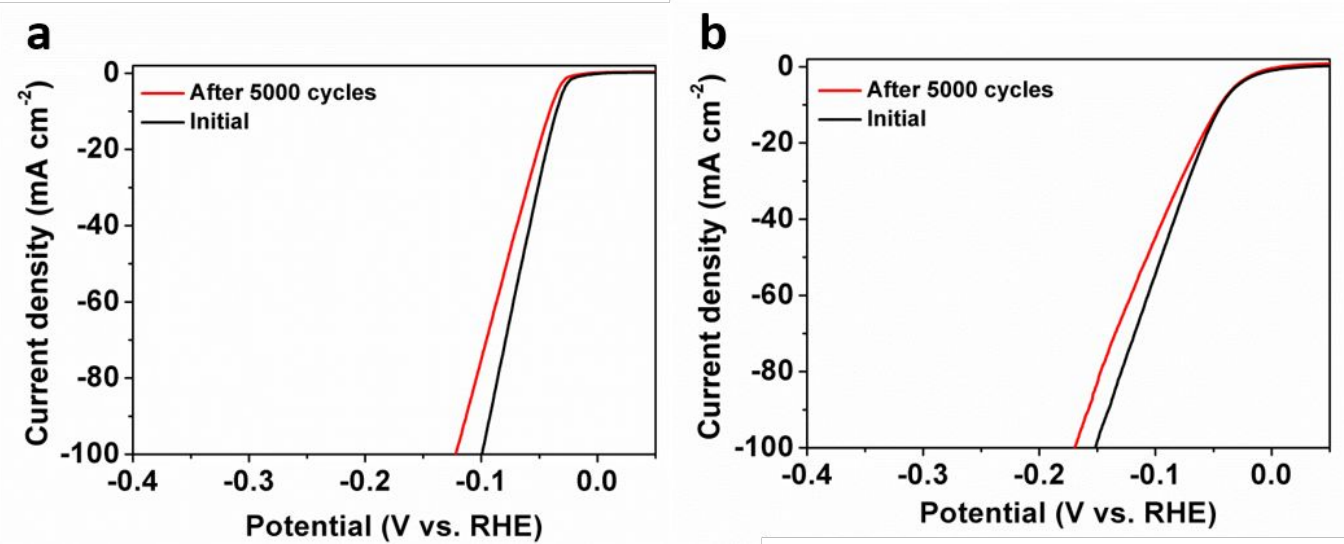

Figure S17 LSVs of (a) Pt/C and (b) $\mathrm{Pt}_{1} / \mathrm{NNGF}$ powder catalysts before and after ADT for 5000 cycles. 


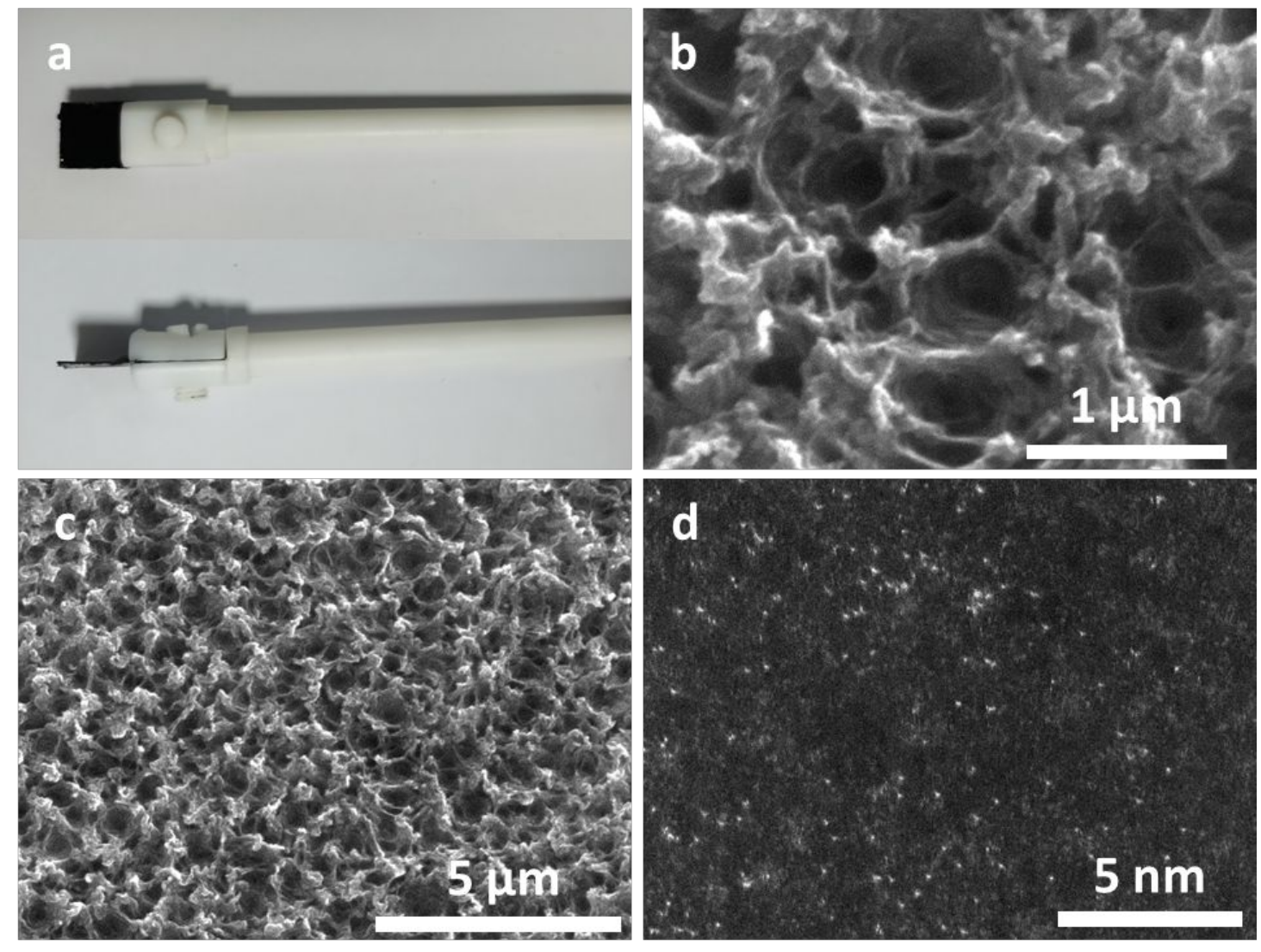

Figure S18 (a) Digital photo, (b, c) SEM and (d) HAADF-STEM images of $\mathrm{Pt}_{1} / \mathrm{NNGF}$ electrode after ADT.

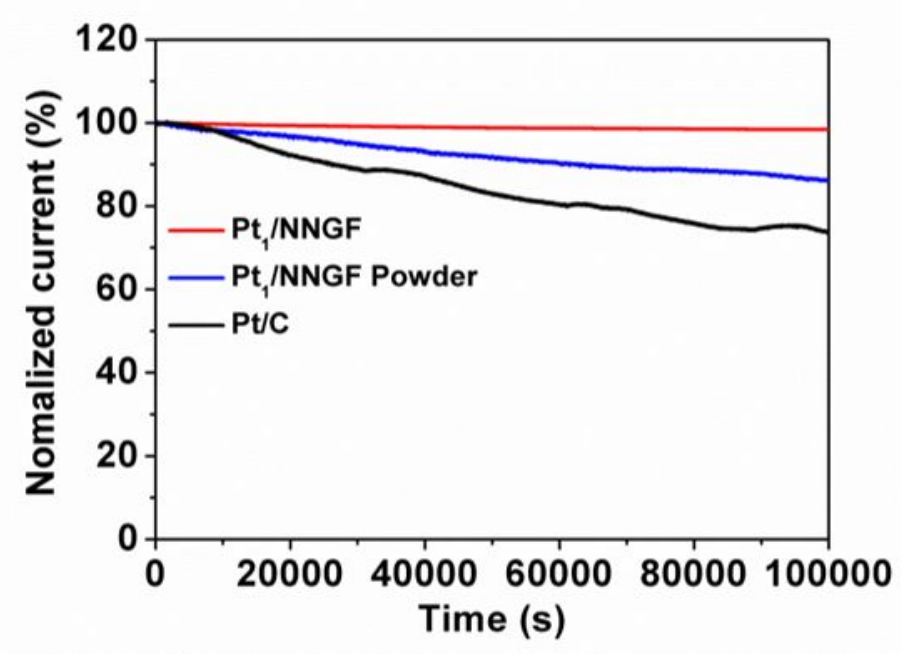

Figure S19 Long-term stability performance of $\mathrm{Pt} / \mathrm{C}, \mathrm{Pt}_{1} / \mathrm{NNGF}$ and $\mathrm{Pt}_{1} / \mathrm{NNGF}$ powder catalysts. 


\section{Supporting Tables}

Table S1. $\mathrm{N}$ contents in $\mathrm{NNGF}$ and $\mathrm{Pt}_{1} / \mathrm{NNGF}$.

\begin{tabular}{ccccc}
\hline Sample & Pyridinic N/Pt-N(\%) & Pyrolic N(\%) & Graphitic N(\%) & Oxidized N(\%) \\
\hline NNGF & 34.1 & 30.5 & 22.9 & 12.5 \\
$\mathrm{Pt}_{1} / \mathrm{NNGF}$ & 43.3 & 39.2 & 16.6 & 0.9 \\
\hline
\end{tabular}

Table S2. EXAFS fitting parameters at the Pt $\mathrm{L}_{\mathrm{III}}$-edge for various samples $\left(S_{0}{ }^{2}=0.831\right)$

\begin{tabular}{ccccccc}
\hline Sample & Shell & $N^{a}$ & $R(\AA)^{b}$ & $\sigma^{2}\left(\AA^{2}\right)^{c}$ & $\Delta E_{0}(\mathrm{eV})^{d}$ & $R$ factor \\
\hline Pt foil & Pt-Pt & 12 & 2.76 & 0.0044 & 6.2 & 0.0015 \\
& $\mathrm{Pt}-\mathrm{O}$ & 6.0 & 2.02 & 0.0027 & & \\
$\mathrm{PtO}_{2}$ & $\mathrm{Pt}-\mathrm{Pt}$ & 11.6 & 3.11 & 0.0054 & 9.6 & 0.0008 \\
& $\mathrm{Pt}-\mathrm{N}$ & 3.8 & 2.03 & 0.0075 & 1.7 & 0.0002 \\
\hline
\end{tabular}

Table S3. HER performance comparison of Pt SAC based catalysts.

\begin{tabular}{|c|c|c|c|c|}
\hline Catalyst & $\eta_{10}(\mathrm{mV})$ & $\eta_{100}(\mathrm{mV})$ & $\begin{array}{c}\text { Tafel slope ( } \mathrm{mV} \mathrm{dec}- \\
1)\end{array}$ & References \\
\hline $\mathrm{Pt}_{1} / \mathrm{AG}$ & 12 & - & 29.3 & 1 \\
\hline $\mathrm{Pt}_{1} / \mathrm{VNGNMAs}$ & 15 & 110 & 49 & 2 \\
\hline $\mathrm{Pt}_{1} /$ graphdiyne & 68 & - & 46.6 & 3 \\
\hline $\mathrm{Pt}_{1} /$ porous carbon & 105 & - & 65.3 & 4 \\
\hline $\mathrm{Pt}_{1} / \mathrm{NPC}$ & 25 & & 28 & 5 \\
\hline $\mathrm{Pt}_{1} / \mathrm{SWNT}$ & 27 & 130 & 38 & 6 \\
\hline $\mathrm{Pt}_{1} / \mathrm{NGN}$ & 39 & - & 29 & 7 \\
\hline $\mathrm{Pt}_{1} / \mathrm{NMC}$ & 28 & 55 & 26 & 8 \\
\hline $\begin{array}{c}\mathrm{Pt}_{1} / \mathrm{CoP} \\
\text { nanotube/Ni foam* }\end{array}$ & 24 & - & 30 & 9 \\
\hline $\mathrm{Pt}_{1} / \mathrm{MoS}_{2}$ & 170 & - & 96 & 10 \\
\hline $\mathrm{Pt}_{1} / \mathrm{NNGF}$ & 23 & 58 & 29.1 & This work \\
\hline
\end{tabular}

*In neutral solution

\section{References}

[1] Ye, S.; Luo, F.; Zhang, Q.; Zhang, P.; Xu, T.; Wang, Q.;He, D.; Guo, L.; Zhang, Y.; He, C.; Ouyang, X.; Gu, M.; Liu, J.; Sun, X. Highly stable single Pt atomic sites anchored on aniline-stacked graphene for hydrogen evolution reaction. Energy Environ. Sci. 2019, 12, 1000-1007.

[2] Chi, K.; Chen, Z.; Xiao, F.; Guo, W.; Xi, W.; Liu, J.; Yan, H.; Zhang, Z.; Xiao, J.; Liu, J.; Luo, J.;Wang, S.; Loh, K. P. Maximizing the utility of single atom electrocatalysts on a 3D graphene nanomesh. J. Mater. Chem. A 2019, 7, 15575-15579.

[3] Yin, X. P.; Wang, H. J.; Tang, S. F.; Lu, X. L.; Shu, M.; Si, R.; Lu, T. B. Engineering the coordination environment of single-atom platinum anchored on graphdiyne for optimizing electrocatalytic hydrogen evolution. Angew. Chem. Int. Ed. 2018, 57, 9382-9386.

[4] Zhang, H.; An, P.; Zhou, W.; Guan, B. Y.; Zhang, P.; Dong, J.; Lou, X. W. Dynamic traction 
of lattice-confined platinum atoms into mesoporous carbon matrix for hydrogen evolution reaction. Sci. Adv. 2018, 4, 6657.

[5] Li, T.; Liu, J.; Song, Y.; Wang, F. Photochemical solid-phase synthesis of platinum single atoms on nitrogen-doped carbon with high loading as bifunctional catalysts for hydrogen evolution and oxygen reduction reactions. ACS Catal. 2018, 8, 8450-8458.

[6] Tavakkoli, M.; Holmberg, N.; Kronberg, R.; Jiang, H.; Sainio, J.; Kauppinen, E. I.; Kallio, T.; Laasonen, K. Electrochemical activation of single-walled carbon nanotubes with pseudo-atomic-scale platinum for the hydrogen evolution reaction. ACS Catal. 2017, 7, 3121-3130.

[7] Cheng, N.; Stambula, S.; Wang, D.; Banis, M. N.; Liu, J.; Riese, A.; Xiao, B.; Li, R.; Sham, T. K.; Liu, L. M.; Botton, G. A.; Sun, X. Platinum single-atom and cluster catalysis of the hydrogen evolution reaction. Nat. Commun. 2016, 7, 13638.

[8] Wei, H.; Wu, H.; Huang, K.; Ge, B.; Ma, J.; Lang, J.; Zu, D.; Lei, M.; Yao, Y.; Guo, W.; Wu, H. Ultralow-temperature photochemical synthesis of atomically dispersed Pt catalysts for the hydrogen evolution reaction. Chem. Sci. 2019, 10, 2830-2836.

[9] Zhang, L.; Han, L.; Liu, H.; Liu, X.; Luo, J. Potential-cycling synthesis of single platinum atoms for efficient hydrogen evolution in neutral media. Angew. Chem. Int. Ed. 2017, 56, 13694-13698.

[10] Deng, J.; Li, H.; Xiao, J.; Tu, Y.; Deng, D.; Yang, H.; Tian, H.; Li, J.; Ren, P.; Bao, X. Triggering the electrocatalytic hydrogen evolution activity of the inert two-dimensional $\mathrm{MoS}_{2}$ surface via single-atom metal doping. Energy Environ. Sci. 2015, 8, 1594-1601. 